\title{
Existence Criteria for Katugampola Fractional Type Impulsive Differential Equations with Inclusions
}

\author{
Murugaiya Janaki ${ }^{1}$, Kuppusamy Kanagarajan ${ }^{1}$ and Elsayed Mohammed Elsayed ${ }^{2,3^{*}}$ \\ ${ }^{1}$ Department of Mathematics, Sri Ramakrishna Mission Vidyalaya College of Arts and Science, Coimbatore-641020, India \\ ${ }^{2}$ Department of Mathematics, King Abdulaziz University, Saudi Arabia \\ ${ }^{3}$ Department of Mathematics, Mansoura University, Egypt \\ * Corresponding author
}

\section{Article Info}

Keywords: Differential inclusion, Existence, Fixed point, Impulses, Katugampola fractional derivative, Uniqueness. 2010 AMS: 26A33, 34A37.

Received: 17 June 2018

Accepted: 20 September 2018

Available online: 20 April 2019

\begin{abstract}
In this paper, we consider the existence and uniqueness of solutions to the impulsive differential equations with inclusions involving Katugampola fractional derivative. With the help of properties of Katugampola fractional calculus and fixed point methods, we derive existence and uniqueness results. Finally, an example is given to illustrate our theoretical results.
\end{abstract}

\section{Introduction}

Fractional calculus and its potential applications have gained a lot of importance, mainly because fractional calculus has become a powerful tool with more accurate and successful results in modelling several complex phenomena in numerous seemingly diverse and widespread fields of science and engineering. Many fields such as physics, fluid mechanics, viscoelasticity, heat conduction in materials with memory, chemistry and engineering can be described by fractional differential equations, see the basic books [1,2,3]. Recently, some basic theory for fractional differential equations and inclusions was discussed see the papers $[4,5,6,7,8,9]$ and the references therein. In recent years, attention has been paid to establish sufficient conditions for the existence results to differential systems involving Katugampola fractional derivatives see the papers $[10,11,12]$. In recent years, numerous contributions have been made in the theory and applications of (impulsive) fractional differential equations. The theory of impulsive differential equations and impulsive differential inclusions has been an object interest because of its wide applications in physics, biology, engineering, medical fields, industry and technology. The reason for this applicability arises from the fact that impulsive differential problems are an appropriate model for describing process which at certain moments change their state rapidly and which cannot be described using the classical differential problems. For some of these applications we refer to $[13,14,15,16,17]$. During the last ten years, impulsive differential inclusions with different condition have intensely studied by many mathematicians. At present, the foundations of the general theory of impulsive differential equations and inclusions are already laid, and many of them are investigated in details in the papers of Benchohra et al. [18, 19].

In this paper we are concerned with the existence of the following Katugampola fractional impulsive differential inclusions of the type,

$$
\begin{cases}\rho^{\rho} D_{0^{+}}^{\omega} u(x) \in H(x, u(x)), & x \in \mathfrak{J}=[0, T], x \neq x_{m}, m=1,2, \ldots k, 1<\omega \leq 2, \\ \left.\Delta u\right|_{x=x_{m}}=I_{m}\left(u\left(x_{m}^{-}\right)\right), & m=1,2, \ldots, k, \\ \left.\Delta u^{\prime}\right|_{x=x_{m}}=\bar{I}_{m}\left(u\left(x_{m}^{-}\right)\right), & m=1,2, \ldots, k, \\ u(0)=u_{0}, u^{\prime}(0)=u_{1}, & \end{cases}
$$

where ${ }^{\rho} D^{\omega}$ is the Katugampola fractional derivative in Caputo sense, $H: \mathfrak{J} \times \mathbb{R} \rightarrow P(\mathbb{R})$ is a multivalued map, $[P(\mathbb{R})$ is the family of all nonempty subset of $\mathbb{R}], I_{m}$ and $\bar{I}_{m}: \mathbb{R} \rightarrow \mathbb{R}, m=1,2, \cdots k$, and $u_{0}, u_{1} \in \mathbb{R}, 0=x_{0}<x_{1}<\cdots<x_{k}<x_{k+1}=T,\left.\Delta u\right|_{x=x_{m}}=u\left(x_{m}^{+}\right)-u\left(x_{m}^{-}\right)$, $\left.\Delta u^{\prime}\right|_{x=x_{m}}=u^{\prime}\left(x_{m}^{+}\right)-u^{\prime}\left(x_{m}^{-}\right), u\left(x_{m}^{+}\right)=\lim _{l \rightarrow 0^{+}} u\left(x_{m}+l\right)$ and $u\left(x_{m}^{-}\right)=\lim _{l \rightarrow 0^{-}} u\left(x_{m}+l\right)$ denotes the right and left limits of $u(x)$ at $x=x_{m}$, $m=1,2, \ldots k$. 


\section{Prerequisites}

In this section, we introduce notations, definitions, lemmas and theorems that will be used for the main results.

Let $\mathfrak{C}(\mathfrak{J}, \mathbb{R})$ be the Banach space of all continuous functions from $\mathfrak{J}$ into $\mathbb{R}$ with the norm

$$
\|u\|_{\infty}=\sup \{|u(x)|: 0 \leq x \leq T\},
$$

and let $\mathfrak{L}^{1}(\mathfrak{J}, \mathbb{R})$ be the Banach space of functions $u: \mathfrak{J} \rightarrow \mathbb{R}$ that are Lebesgue integrable with the norm

$$
\|u\|_{\mathfrak{L}^{1}}=\int_{0}^{T}|u(x)| \mathrm{d} x .
$$

The space $\mathfrak{A} \mathfrak{C}^{-1}(\mathfrak{J}, \mathbb{R})$ consists of functions $u: \mathfrak{J} \rightarrow \mathbb{R}$, which are absolutely continuous, whose first derivative $u^{\prime}$ is absolutely continuous. Let $(Y,\|\cdot\|)$ be a Banach space and let us assume that

$$
\begin{gathered}
Q_{c l}(Y)=\{X \in P(Y): X \text { closed }\}, \\
Q_{b}(Y)=\{X \in P(Y): X \text { bounded }\}, \\
Q_{c p}(Y)=\{X \in P(Y): X \text { compact }\},
\end{gathered}
$$

and,

$$
Q_{c p, c}(Y)=\{X \in P(Y): X \text { compact and convex }\}
$$

A multivalued map $F: Y \rightarrow Q(Y)$ is convex(closed) valued if $F(t)$ is convex(closed) for all $t \in Y$. $F$ is bounded on bounded sets if $F(B)=\cup_{t \in B} F(t)$ is bounded in $Y$ for all $B \in Q_{b}(Y)$ (i.e., $\sup _{t \in B}\{\sup \{|u|: u \in F(t)\}\}<\infty$ ). $F$ is called upper semi-continuous on $Y$ if for each $t_{0} \in Y$, the set $F\left(t_{0}\right)$ is a nonempty closed subset of $Y$, and if for each open set $M$ of $Y$ containing $F\left(t_{0}\right)$, there exists an open neighborhood $M_{0}$ of $t_{0}$ such that $F\left(M_{0}\right) \subseteq M$. F is said to be completely continuous if $F(\mathscr{B})$ is relatively compact for every $\mathscr{B} \in Q_{b}(Y)$. If the multivalued map $F$ is completely continuous with nonempty compact values, then $F$ is upper semi-continuous if and only if $F$ has a closed graph (i.e., $t_{n} \rightarrow t_{*}, u_{n} \rightarrow u_{*}, u_{n} \in F\left(t_{n}\right) \Rightarrow u_{*} \in F\left(t_{*}\right)$ ). $F$ has a fixed point if there is $t \in Y$ such that $t \in F(t)$.

The fixed point set of the multivalued operator $F$ will be denoted by Fix $F$. A multivalued map $F: \mathfrak{J} \rightarrow Q_{c l}(\mathbb{R})$ is said to be measurable if for every $u \in \mathbb{R}$, the function

$$
x \mapsto d(u, F(x))=\inf \{|u-w|: w \in F(x)\},
$$

is measurable. See the books of Aubin and Cellina [5], Deimling [20] and Hu and Papageorgiou [21] for more details.

Definition 2.1. A multivalued map $H: \mathfrak{J} \times \mathbb{R} \rightarrow P(\mathbb{R})$ is said to be Carathéodory if

(i) $x \mapsto H(x, y)$ is measurable for each $y \in \mathbb{R}$;

(ii) $y \mapsto H(x, y)$ is upper semi-continuous for almost all $x \in \mathfrak{J}$.

For each $u \in \mathfrak{P C}(\mathfrak{J}, \mathbb{R})$, define the set of selections of $H$ by

$$
S_{H, u}=\left\{g \in \mathfrak{L}^{1}(\mathfrak{J}, \mathbb{R}): g(x) \in H(x, u(x)) \text { a.e. } x \in \mathfrak{J}\right\} .
$$

Let $(Y, d)$ be a metric space induced from the normed space $(Y,|\cdot|)$. Consider $G_{d}: P(Y) \times P(Y) \rightarrow \mathbb{R}_{+} \cup\{\infty\}$ given by

$$
F_{d}(A, B)=\max \left\{\sup _{a \in A} d(a, B), \sup _{b \in B} d(A, b)\right\},
$$

where $d(A, b)=\inf _{a \in A} d(a, b), d(a, B)=\inf _{b \in B} d(a, b)$. Then $\left(Q_{b, c l}(Y), G_{d}\right)$ is a metric space and $\left(Q_{c l}(Y), G_{d}\right)$ is a generalized metric space. See the paper [22].

Definition 2.2. A multivalued operator $M: Y \rightarrow Q_{c l}(Y)$ is called

(a) $\gamma$-Lipschitz, if and only if there exists $\gamma>0$ such that

$$
G_{d}(M(t), M(u)) \leq \gamma d(t, u), \text { for each } t, u \in Y,
$$

(b) a contraction if and only if it is $\gamma$-Lipschitz with $\gamma<1$.

Lemma 2.3. [23] Let $(Y, d)$ be a complete metric space. If $M: Y \rightarrow Q_{c l}(Y)$ is a contraction, then Fix $M \neq \emptyset$.

Definition 2.4. [2, 3] The fractional(arbitrary) order integral of the function $h \in \mathfrak{L}^{1}\left([a, b], \mathbb{R}_{+}\right)$of order $\omega \in \mathbb{R}_{+}$is defined by

$$
I_{a}^{\omega} h(x)=\frac{1}{\Gamma(\omega)} \int_{a}^{x}(x-s)^{\omega-1} h(s) \mathrm{d} s
$$

where $\Gamma$ is the gamma function. When $a=0$, we write $I^{\omega} h(x)=h(x) * \psi_{\omega}(x)$, where $\psi_{\omega}(x)=\frac{x^{\omega-1}}{\Gamma(\omega)}$ for $x>0$, and $\psi_{\omega}(x)=0$ for $x \leq 0$, and $\psi_{\omega} \rightarrow \delta(x)$ as $\omega \rightarrow 0$, where $\delta$ is the delta function. 
Definition 2.5. [2, 3] For a function $h$ given on the interval $[a, b]$, the Caputo fractional-order derivative of $h$, is defined by

$$
\left({ }^{c} D_{a^{+}}^{\omega} h\right)(x)=\frac{1}{\Gamma(n-\omega)} \int_{a}^{x}(x-s)^{n-\omega-1} h^{(n)}(s) \mathrm{d} s,
$$

where $n=[\omega]+1$ and $[\omega]$ denotes the integer part of the real number $\omega$.

Now, we consider the definitions of the generalized fractional operators introduced in $[10,11,12]$.

Definition 2.6. The generalized left-sided fractional integral ${ }^{\rho} I_{a^{+}}^{\omega} h$ of order $\omega \in \mathbb{C}(\operatorname{Re}(\omega)>0)$ is defined by

$$
\left({ }^{\rho} I_{a^{+}}^{\omega} h\right)(x)=\frac{\rho^{1-\omega}}{\Gamma(\omega)} \int_{a}^{x}\left(x^{\rho}-s^{\rho}\right)^{\omega-1} s^{\rho-1} h(s) \mathrm{d} s,
$$

for $x>a$, if the integral exists.

Definition 2.7. The generalized fractional derivative, corresponding to the generalized fractional integral (2.1), is defined for $x>a$, by

$$
\begin{aligned}
\left({ }^{\rho} D_{a^{+}}^{\omega} h\right)(x) & =\left(x^{1-\rho} \frac{\mathrm{d}}{\mathrm{d} x}\right)^{n}\left({ }^{\rho} I_{a^{+}}^{n-\omega} h\right)(x) \\
& =\frac{\rho^{\omega-n+1}}{\Gamma(n-\omega)}\left(x^{1-\rho} \frac{\mathrm{d}}{\mathrm{d} x}\right)^{n} \int_{a}^{x}\left(x^{\rho}-s^{\rho}\right)^{n-\omega-1} s^{\rho-1} h(s) \mathrm{d} s
\end{aligned}
$$

if the integral exists.

Definition 2.8. The Caputo-type generalized fractional derivative, ${ }_{c}^{\rho} D_{a^{+}}^{\omega}$ is defined via the above generalized fractional derivative (2.2) as follows

$$
{ }_{c}^{\rho} D_{a^{+}}^{\omega} h(x)=\left({ }^{\rho} D_{a^{+}}^{\omega}\left[h(s)-\sum_{m=0}^{n-1} \frac{h^{(m)}(a)}{m !}(s-a)^{m}\right]\right)(x),
$$

where $n=\lceil\operatorname{Re}(\omega)\rceil$.

Sufficient conditions are given in [2] for the fractional differential and integral to exist.

\section{The convex case}

In this section, we discuss about the existence of solutions for the problem (1.1) when the right hand side has the convex values. For this, we assume that $H$ is a compact, convex valued and multivalued map. Consider the Banach space,

$$
\begin{gathered}
\mathfrak{P C}(\mathfrak{J}, \mathbb{R})=\left\{u: \mathfrak{J} \rightarrow \mathbb{R}: u \in \mathfrak{C}\left(\left(x_{m}, x_{m+1}\right], \mathbb{R}\right), m=0,1, \ldots k+1\right. \\
\text { and there exist } \left.u\left(x_{m}^{-}\right), u\left(x_{m}^{+}\right), m=1,2, \ldots k \text { with } u\left(x_{m}^{-}\right)=u\left(x_{m}\right)\right\},
\end{gathered}
$$

with the norm

$$
\|u\|_{\mathfrak{P C}}=\sup _{x \in \mathfrak{J}}|u(x)|
$$

Set $\mathfrak{J}^{\prime}:=[0, T] \backslash\left\{x_{1}, x_{2}, \ldots x_{k}\right\}$.

Definition 3.1. A function $u \in \mathfrak{P C}(\mathfrak{J}, \mathbb{R}) \cap \cup_{m=0}^{k} \mathfrak{A} \mathfrak{C}^{1}\left(\left(x_{m}, x_{m+1}\right), \mathbb{R}\right)$ with its $\omega$-derivative exists on $\mathfrak{J}^{\prime}$ is said to be a solution of (1.1), if there exists a function $g \in \mathfrak{L}^{1}([0, T], \mathbb{R})$ such that $g(x) \in H(x, u(x))$ a.e. $x \in \mathfrak{J}$ satisfies the differential equation ${ }^{\rho} D_{0^{+}}^{\omega} u(x)=g(x)$ on $\mathfrak{J}^{\prime}$, and conditions

$$
\begin{gathered}
\left.\Delta u\right|_{x=x_{m}}=I_{m}\left(u\left(x_{m}^{-}\right)\right), m=1,2, \ldots k, \\
\left.\Delta u^{\prime}\right|_{x=x_{m}}=\bar{I}_{m}\left(u\left(x_{m}^{-}\right)\right), m=1,2, \ldots k, \\
u(0)=u_{0}, u^{\prime}(0)=u_{1},
\end{gathered}
$$

are satisfied. Let $h:[a, b] \rightarrow \mathbb{R}$ be a continuous function. We need the following lemmas for the existence of solutions for the problem (1.1).

Lemma 3.2. [9] Let $\omega>0$, then the differential equation

$$
{ }^{\rho} D_{0^{+}}^{\omega} h(x)=0
$$

has solutions $h(x)=b_{0}+b_{1}\left(\frac{x^{\rho}}{\rho}\right)+b_{2}\left(\frac{x^{\rho}}{\rho}\right)^{2}+\cdots+b_{n-1}\left(\frac{x^{\rho}}{\rho}\right)^{(n-1)}, b_{i} \in \mathbb{R}, i=0,1,2, \ldots, n-1, n=[\omega]+1$.

Lemma 3.3. [9] Let $\omega>0$, then

$$
{ }^{\rho} I_{0^{+}}^{\omega}\left({ }^{\rho} D_{0^{+}}^{\omega} h(x)\right)=h(x)+b_{0}+b_{1}\left(\frac{x^{\rho}}{\rho}\right)+b_{2}\left(\frac{x^{\rho}}{\rho}\right)^{2}+\cdots+b_{n-1}\left(\frac{x^{\rho}}{\rho}\right)^{(n-1)},
$$

for some $b_{i} \in \mathbb{R}, i=0,1,2, \ldots, n-1, n=[\omega]+1$.

From Lemma 3.2, and 3.3, we get the following results which is useful in the following sequel. 
Lemma 3.4. Let $1<\omega \leq 2$ and let $g \in \mathfrak{P C}(\mathfrak{J}, \mathbb{R})$. A function $u$ is a solution of the fractional integral equation

$$
u(x)= \begin{cases}u_{0}+u_{1}\left(\frac{x^{\rho}}{\rho}\right)+\frac{\rho^{1-\omega}}{\Gamma(\omega)} \int_{0}^{x}\left(x^{\rho}-s^{\rho}\right)^{\omega-1} s^{\rho-1} g(s) \mathrm{d} s, & \text { if } x \in\left[0, x_{1}\right], \\ u_{0}+u_{1}\left(\frac{x^{\rho}}{\rho}\right)+\frac{\rho^{1-\omega}}{\Gamma(\omega)} \sum_{i=1}^{m} \int_{x_{i-1}}^{x_{i}}\left(x_{i}^{\rho}-s^{\rho}\right)^{\omega-1} s^{\rho-1} g(s) \mathrm{d} s & \\ +\frac{\rho^{2-\omega}}{\Gamma(\omega-1)} \sum_{i=1}^{m}\left(x^{\rho-1}-x_{i}^{\rho-1}\right) \int_{x_{i-1}}^{x_{i}}\left(x_{i}^{\rho}-s^{\rho}\right)^{\omega-2} s^{\rho-1} g(s) \mathrm{d} s & \\ +\frac{\rho^{1-\omega}}{\Gamma(\omega)} \int_{x_{m}}^{x}\left(x^{\rho}-s^{\rho}\right)^{\omega-1} s^{\rho-1} g(s) \mathrm{d} s & \text { if } x \in\left(x_{m}, x_{m+1}\right], m=1,2, \ldots k, \\ +\sum_{i=1}^{m} I_{i}\left(u\left(x_{i}^{-}\right)\right)+\sum_{i=1}^{m}\left(x-x_{i}\right) \bar{I}_{i}\left(u\left(x_{i}^{-}\right)\right), & \end{cases}
$$

if and only if $u$ is a solution of the fractional initial value problem

$$
\begin{cases}\rho^{\rho} D_{0^{+}}^{\omega} u(x)=g(x), & \text { for each } x \in \mathfrak{J}^{\prime}, \\ \left.\Delta u\right|_{x=x_{m}}=I_{m}\left(u\left(x_{m}^{-}\right)\right), & m=1,2, \ldots, k \\ \left.\Delta u^{\prime}\right|_{x=x_{m}}=\bar{I}_{m}\left(u\left(x_{m}^{-}\right)\right), & m=1,2, \ldots, k \\ u(0)=u_{0}, u^{\prime}(0)=u_{1} . & \end{cases}
$$

Proof. Assume that $u$ satisfies (3.2). If $x \in\left[0, x_{1}\right]$ then ${ }^{\rho} D_{0^{+}}^{\omega} u(x)=g(x)$. From Lemma 3.3, we get

$$
u(x)=b_{0}+b_{1}\left(\frac{x^{\rho}}{\rho}\right)+\frac{\rho^{1-\omega}}{\Gamma(\omega)} \int_{0}^{x}\left(x^{\rho}-s^{\rho}\right)^{\omega-1} s^{\rho-1} g(s) \mathrm{d} s .
$$

Hence $b_{0}=u_{0}, b_{1}=u_{1}$. Thus

$$
u(x)=u_{0}+u_{1}\left(\frac{x^{\rho}}{\rho}\right)+\frac{\rho^{1-\omega}}{\Gamma(\omega)} \int_{0}^{x}\left(x^{\rho}-s^{\rho}\right)^{\omega-1} s^{\rho-1} g(s) \mathrm{d} s .
$$

If $x \in\left(x_{1}, x_{2}\right]$, then from Lemma 3.3, we arrive to

$$
\begin{aligned}
u(x)= & b_{0}+b_{1}\left(\frac{x^{\rho}-x_{1}^{\rho}}{\rho}\right)+\frac{\rho^{1-\omega}}{\Gamma(\omega)} \int_{x_{1}}^{x}\left(x^{\rho}-s^{\rho}\right)^{\omega-1} s^{\rho-1} g(s) \mathrm{d} s . \\
\left.\Delta u\right|_{x=x_{1}} & =u\left(x_{1}^{+}\right)-u\left(x_{1}^{-}\right) \\
& =b_{0}-\left(u_{0}+u_{1}\left(\frac{x_{1}^{\rho}}{\rho}\right)+\frac{\rho^{1-\omega}}{\Gamma(\omega)} \int_{0}^{x_{1}}\left(x_{1}^{\rho}-s^{\rho}\right)^{\omega-1} s^{\rho-1} g(s) \mathrm{d} s\right) \\
& =I_{1}\left(u\left(x_{1}^{-}\right)\right) .
\end{aligned}
$$

Hence,

$$
\begin{aligned}
b_{0}=u_{0} & +u_{1}\left(\frac{x_{1}^{\rho}}{\rho}\right)+\frac{\rho^{1-\omega}}{\Gamma(\omega)} \int_{0}^{x_{1}}\left(x_{1}^{\rho}-s^{\rho}\right)^{\omega-1} s^{\rho-1} g(s) \mathrm{d} s+I_{1}\left(u\left(x_{1}^{-}\right)\right) . \\
\left.\Delta u^{\prime}\right|_{x=x_{1}} & =u^{\prime}\left(x_{1}^{+}\right)-u^{\prime}\left(x_{1}^{-}\right) \\
& =b_{1}-\left(u_{1}+\frac{\rho^{2-\omega}\left(x^{\rho-1}-x_{1}^{\rho-1}\right)}{\Gamma(\omega-1)} \int_{0}^{x_{1}}\left(x_{1}^{\rho}-s^{\rho}\right)^{\omega-2} s^{\rho-1} g(s) \mathrm{d} s\right) \\
& =\bar{I}_{1}\left(u\left(x_{1}^{-}\right)\right),
\end{aligned}
$$

and

$$
b_{1}=u_{1}+\frac{\rho^{2-\omega}}{\Gamma(\omega-1)}\left(x^{\rho-1}-x_{1}^{\rho-1}\right) \int_{0}^{x_{1}}\left(x_{1}^{\rho}-s^{\rho}\right)^{\omega-2} s^{\rho-1} g(s) \mathrm{d} s+\bar{I}_{1}\left(u\left(x_{1}^{-}\right)\right) .
$$

Then by (3.3)-(3.5), we get

$$
\begin{aligned}
u(x) & =u_{0}+u_{1}\left(\frac{x^{\rho}}{\rho}\right)+\frac{\rho^{1-\omega}}{\Gamma(\omega)} \int_{0}^{x_{1}}\left(x_{1}^{\rho}-s^{\rho}\right)^{\omega-1} s^{\rho-1} g(s) \mathrm{d} s \\
& +\frac{\rho^{2-\omega}}{\Gamma(\omega-1)}\left(x^{\rho-1}-x_{1}^{\rho-1}\right) \int_{0}^{x_{1}}\left(x_{1}^{\rho}-s^{\rho}\right)^{\omega-2} s^{\rho-1} g(s) \mathrm{d} s \\
& +\frac{\rho^{1-\omega}}{\Gamma(\omega)} \int_{x_{1}}^{x}\left(x^{\rho}-s^{\rho}\right)^{\omega-1} s^{\rho-1} g(s) \mathrm{d} s \\
& +I_{1}\left(u\left(x_{1}^{-}\right)\right)+\left(x-x_{1}\right) \bar{I}_{1}\left(u\left(x_{1}^{-}\right)\right) .
\end{aligned}
$$

If $x \in\left(x_{m}, x_{m+1}\right]$, then from Lemma 3.3, we get (3.1). Conversely, assume that $u$ satisfies the equation (3.1). If $x \in\left[0, x_{1}\right]$ then $u(0)=u_{0}$, $u^{\prime}(0)=u_{1}$ and using the concept that ${ }^{\rho} D_{0^{+}}^{\omega}$ is the left inverse of ${ }^{\rho} I_{0^{+}}^{\omega}$, we get,

$$
{ }^{\rho} D_{0^{+}}^{\omega} u(x)=g(x), \text { for each } x \in\left[0, x_{1}\right] .
$$


If $x \in\left[x_{m}, x_{m+1}\right), m=1,2, \ldots k$ and using the fact that ${ }^{\rho} D_{0^{+}}^{\omega} L=0$, where $L$ is a constant, we have

$$
{ }^{\rho} D_{0^{+}}^{\omega} u(x)=g(x), \text { for each } x \in\left[x_{m}, x_{m+1}\right) .
$$

Also, we can easily prove that

$$
\begin{gathered}
\left.\Delta u\right|_{x=x_{m}}=I_{m}\left(u\left(x_{m}^{-}\right)\right), m=1,2, \ldots, k, \\
\left.\Delta u^{\prime}\right|_{x=x_{m}}=\bar{I}_{m}\left(u\left(x_{m}^{-}\right)\right), m=1,2, \ldots, k .
\end{gathered}
$$

By using the nonlinear alternative of Leray-Schauder type for multivalued maps [24], we can prove our first result. For this, we assume the following hypotheses:

(A1) $H: \mathfrak{J} \times \mathbb{R} \rightarrow P_{c p, c}(\mathbb{R})$ is a Carathéodory multivalued map.

(A2) There exists $q \in \mathfrak{C}\left(\mathfrak{J}, \mathbb{R}^{+}\right)$and $\Phi:[0, \infty) \rightarrow(0, \infty)$ continuous and nondecreasing such that

$$
\|H(x, y)\|_{P}=\sup \{|g|: g \in H(x, y)\} \leq q(x) \Phi(|y|) \text {, for } x \in \mathfrak{J} \text { and } y \in \mathbb{R} .
$$

(A3) There exist $\Phi^{*}, \bar{\Phi}^{*}:[0, \infty) \rightarrow(0, \infty)$ continuous and nondecreasing such that

$$
\begin{gathered}
\left|I_{m}(y)\right| \leq \Phi^{*}(|y|), \text { for } y \in \mathbb{R} \\
\left|\bar{I}_{m}(y)\right| \leq \bar{\Phi}^{*}(|y|), \text { for } y \in \mathbb{R} .
\end{gathered}
$$

(A4) There exists a number $N>0$ such that

$$
\frac{N}{\left|u_{0}\right|+T^{\rho}\left|\frac{u_{1}}{\rho}\right|+a \Phi(N)+k \bar{\Phi}^{*}(N)+k T^{\rho} \bar{\Phi}^{*}(N)}>1,
$$

where $q^{0}=\sup \{q(x): x \in \mathfrak{J}\}$ and $a=\frac{k T^{\rho \omega} q^{0}}{\rho^{\omega} \Gamma(\omega+1)}+\frac{k T^{\rho \omega} q^{0}}{\rho^{\omega-1} \Gamma(\omega)}+\frac{T^{\rho \omega} q^{0}}{\rho^{\omega} \Gamma(\omega+1)}$.

(A5) There exists $\tilde{l} \in \mathfrak{L}^{1}\left(\mathfrak{J}, \mathbb{R}^{+}\right)$such that

$$
G_{d}(H(x, y), H(x, \bar{y})) \leq \tilde{l}(x)|y-\bar{y}| \text { for a.e. } x \in \mathfrak{J}, y, \bar{y} \in \mathbb{R},
$$

$d(0, H(x, 0)) \leq \tilde{l}(x)$, a.e. $x \in \mathfrak{J}$

Theorem 3.5. Under assumptions (A1)-(A5), the initial value problem (1.1) has at least one solution on $\mathfrak{J}$.

Proof. We transform the problem (1.1) into a fixed point problem. Consider the multivalued operator

$$
\begin{aligned}
M(u) & =\left\{h \in \mathfrak{P C}(\mathfrak{J}, \mathbb{R}): h(x)=u_{0}+u_{1}\left(\frac{x^{\rho}}{\rho}\right)+\frac{\rho^{1-\omega}}{\Gamma(\omega)} \sum_{0<x_{m}<x} \int_{x_{m-1}}^{x_{m}}\left(x_{m}^{\rho}-s^{\rho}\right)^{\omega-1} s^{\rho-1} g(s) \mathrm{d} s\right. \\
& +\frac{\rho^{2-\omega}}{\Gamma(\omega-1)} \sum_{0<x_{m}<x}\left(x^{\rho-1}-x_{m}^{\rho-1}\right) \int_{x_{m-1}}^{x_{m}}\left(x_{m}^{\rho}-s^{\rho}\right)^{\omega-2} s^{\rho-1} g(s) \mathrm{d} s \\
& +\frac{\rho^{1-\omega}}{\Gamma(\omega)} \int_{x_{m}}^{x}\left(x^{\rho}-s^{\rho}\right)^{\omega-1} s^{\rho-1} g(s) \mathrm{d} s \\
& \left.+\sum_{0<x_{m}<x} I_{m}\left(u\left(x_{m}^{-}\right)\right)+\sum_{0<x_{m}<x}\left(x-x_{m}\right) \bar{I}_{m}\left(u\left(x_{m}^{-}\right)\right), g \in S_{H, u}\right\} .
\end{aligned}
$$

Clearly from Lemma 3.4, fixed points of $M$ are solutions to (1.1). We shall prove that $M$ satisfies the assumptions of the nonlinear alternative of Leray-Schauder type [24]. The proof of the theorem contains five steps.

Step 1: $M(u)$ is convex for each $u \in \mathfrak{P C}(\mathfrak{J}, \mathbb{R})$. If $h_{1}, h_{2} \in M(u)$, then there exist $g_{1}, g_{2} \in S_{H, u}$ such that for each $x \in \mathfrak{J}$, we obtain,

$$
\begin{aligned}
h_{i}(x) & =u_{0}+u_{1}\left(\frac{x^{\rho}}{\rho}\right)+\frac{\rho^{1-\omega}}{\Gamma(\omega)} \sum_{0<x_{m}<x} \int_{x_{m-1}}^{x_{m}}\left(x_{m}^{\rho}-s^{\rho}\right)^{\omega-1} s^{\rho-1} g_{i}(s) \mathrm{d} s \\
& +\frac{\rho^{2-\omega}}{\Gamma(\omega-1)} \sum_{0<x_{m}<x}\left(x^{\rho-1}-x_{m}^{\rho-1}\right) \int_{x_{m-1}}^{x_{m}}\left(x_{m}^{\rho}-s^{\rho}\right)^{\omega-2} s^{\rho-1} g_{i}(s) \mathrm{d} s \\
& +\frac{\rho^{1-\omega}}{\Gamma(\omega)} \int_{x_{m}}^{x}\left(x^{\rho}-s^{\rho}\right)^{\omega-1} s^{\rho-1} g_{i}(s) \mathrm{d} s \\
& +\sum_{0<x_{m}<x} I_{m}\left(u\left(x_{m}^{-}\right)\right)+\sum_{0<x_{m}<x}\left(x-x_{m}\right) \bar{I}_{m}\left(u\left(x_{m}^{-}\right)\right), i=1,2 .
\end{aligned}
$$


Let $0 \leq d \leq 1$, then for each $x \in \mathfrak{J}$, we have

$$
\begin{aligned}
\left(d h_{1}+(1-d) h_{2}\right)(x) & =u_{0}+u_{1}\left(\frac{x^{\rho}}{\rho}\right)+\frac{\rho^{1-\omega}}{\Gamma(\omega)} \sum_{0<x_{m}<x} \int_{x_{m-1}}^{x_{m}}\left(x_{m}^{\rho}-s^{\rho}\right)^{\omega-1} s^{\rho-1}\left[d g_{1}(s)+(1-d) g_{2}(s)\right] \mathrm{d} s \\
& +\frac{\rho^{2-\omega}}{\Gamma(\omega-1)} \sum_{0<x_{m}<x}\left(x^{\rho-1}-x_{m}^{\rho-1}\right) \int_{x_{m-1}}^{x_{m}}\left(x_{m}^{\rho}-s^{\rho}\right)^{\omega-2} s^{\rho-1}\left[d g_{1}(s)+(1-d) g_{2}(s)\right] \mathrm{d} s \\
& +\frac{\rho^{1-\omega}}{\Gamma(\omega)} \int_{x_{m}}^{x}\left(x^{\rho}-s^{\rho}\right)^{\omega-1} s^{\rho-1}\left[d g_{1}(s)+(1-d) g_{2}(s)\right] \mathrm{d} s \\
& +\sum_{0<x_{m}<x} I_{m}\left(u\left(x_{m}^{-}\right)\right)+\sum_{0<x_{m}<x}\left(x-x_{m}\right) \bar{I}_{m}\left(u\left(x_{m}^{-}\right)\right) .
\end{aligned}
$$

Since $S_{H, u}$ is convex(because $H$ has convex values), we get

$$
d h_{1}+(1-d) h_{2} \in M(u)
$$

Step 2: $M$ maps bounded sets into bounded sets in $\mathfrak{P C}(\mathfrak{J}, \mathbb{R})$. Let $B_{\Omega^{*}}=\left\{u \in \mathfrak{P C}(\mathfrak{J}, \mathbb{R}):\|u\|_{\infty} \leq \Omega^{*}\right\}$ be bounded set in $\mathfrak{P C}(\mathfrak{J}, \mathbb{R})$ and $u \in B_{\Omega^{*}}$. Then for each $h \in M(u)$ and $x \in \mathfrak{J}$, we get $(A 2)-(A 3)$,

$$
\begin{aligned}
|h(x)| & \leq\left|u_{0}\right|+\left|\frac{u_{1}}{\rho}\right| T^{\rho}+\frac{\rho^{1-\omega}}{\Gamma(\omega)} \sum_{0<x_{m}<x} \int_{x_{m-1}}^{x_{m}}\left(x_{m}^{\rho}-s^{\rho}\right)^{\omega-1} s^{\rho-1}|g(s)| \mathrm{d} s \\
& +\frac{\rho^{2-\omega}}{\Gamma(\omega-1)} \sum_{0<x_{m}<x}\left(x^{\rho-1}-x_{m}^{\rho-1}\right) \int_{x_{m-1}}^{x_{m}}\left(x_{m}^{\rho}-s^{\rho}\right)^{\omega-2} s^{\rho-1}|g(s)| \mathrm{d} s \\
& +\frac{\rho^{1-\omega}}{\Gamma(\omega)} \int_{x_{m}}^{x}\left(x^{\rho}-s^{\rho}\right)^{\omega-1} s^{\rho-1}|g(s)| \mathrm{d} s \\
& +\sum_{0<x_{m}<x}\left|I_{m}\left(u\left(x_{m}^{-}\right)\right)\right|+\sum_{0<x_{m}<x}\left(x-x_{m}\right)\left|\bar{I}_{m}\left(u\left(x_{m}^{-}\right)\right)\right| \\
& \leq\left|u_{0}\right|+\left|\frac{u_{1}}{\rho}\right| T^{\rho}+\frac{k T^{\rho \omega} q^{0}}{\rho^{\omega} \Gamma(\omega+1)} \Phi\left(\Omega^{*}\right)+\frac{T^{\rho \omega} q^{0}}{\rho^{\omega-1} \Gamma(\omega)} \Phi\left(\Omega^{*}\right)+\frac{T^{\rho \omega} q^{0}}{\rho^{\omega} \Gamma(\omega+1)} \Phi\left(\Omega^{*}\right)+k \Phi^{*}\left(\Omega^{*}\right)+k \bar{\Phi}^{*}\left(\Omega^{*}\right) .
\end{aligned}
$$

Thus,

$$
\|h\|_{\infty} \leq\left|u_{0}\right|+\left|\frac{u_{1}}{\rho}\right| T^{\rho}+\frac{k T^{\rho \omega} q^{0}}{\rho^{\omega} \Gamma(\omega+1)} \Phi\left(\Omega^{*}\right)+\frac{T^{\rho \omega} q^{0}}{\rho^{\omega-1} \Gamma(\omega)} \Phi\left(\Omega^{*}\right)+\frac{T^{\rho \omega} q^{0}}{\rho^{\omega} \Gamma(\omega+1)} \Phi\left(\Omega^{*}\right)+k \Phi^{*}\left(\Omega^{*}\right)+k \bar{\Phi}^{*}\left(\Omega^{*}\right):=\tilde{\ell}
$$

Step 3: $M$ maps bounded sets into equicontinuous sets of $\mathfrak{P C}(\mathfrak{J}, \mathbb{R})$. Let $t_{1}, t_{2} \in \mathfrak{J}, t_{1}<t_{2}, B_{\Omega^{*}}$ be a bounded $\operatorname{set}$ of $\mathfrak{P C}(\mathfrak{J}, \mathbb{R})$ as in $\operatorname{Step} 2$, let $u \in B_{\Omega^{*}}$ and $h \in M(u)$, then

$$
\begin{aligned}
\left|h\left(t_{2}\right)-h\left(t_{1}\right)\right| & \leq\left|\frac{u_{1}}{\rho}\right|\left(t_{2}^{\rho}-t_{1}^{\rho}\right)+\frac{\rho^{1-\omega}}{\Gamma(\omega)} \sum_{0<x_{m}<t_{2}-t_{1}} \int_{x_{m-1}}^{x_{m}}\left(x_{m}^{\rho}-s^{\rho}\right)^{\omega-1} s^{\rho-1}|g(s)| \mathrm{d} s \\
& +\frac{\rho^{1-\omega}}{\Gamma(\omega)} \int_{x_{m}}^{t_{1}}\left|\left(t_{2}^{\rho}-s^{\rho}\right)^{\omega-1}-\left(t_{1}^{\rho}-s^{\rho}\right)^{\omega-1}\right|\left|s^{\rho-1}\right||g(s)| \mathrm{d} s \\
& +\frac{\rho^{1-\omega}}{\Gamma(\omega)} \int_{t_{1}}^{t_{2}}\left|\left(t_{2}^{\rho}-s^{\rho}\right)^{\omega-1}\right|\left|s^{\rho-1}\right||g(s)| \mathrm{d} s \\
& +\frac{\rho^{2-\omega}}{\Gamma(\omega-1)} \sum_{0<x_{m}<t_{2}-t_{1}}\left(t_{2}^{\rho-1}-x_{m}^{\rho-1}\right) \int_{x_{m-1}}^{x_{m}}\left(x_{m}^{\rho}-s^{\rho}\right)^{\omega-2}\left|s^{\rho-1}\right||g(s)| \mathrm{d} s \\
& +\frac{\rho^{2-\omega}}{\Gamma(\omega-1)} \sum_{0<x_{m}<t_{1}}\left(t_{2}^{\rho-1}-t_{1}^{\rho-1}\right) \int_{x_{m-1}}^{x_{m}}\left(x_{m}^{\rho}-s^{\rho}\right)^{\omega-2}\left|s^{\rho-1}\right||g(s)| \mathrm{d} s \\
& +\sum_{0<x_{m}<t_{2}-t_{1}}\left|I_{m}\left(u\left(x_{m}^{-}\right)\right)\right|+\sum_{0<x_{m}<t_{2}-t_{1}}\left(t_{2}-x_{m}\right)\left|\bar{I}_{m}\left(u\left(x_{m}^{-}\right)\right)\right| \\
& +\sum_{0<x_{m}<t_{1}}\left(t_{2}-t_{1}\right)\left|\bar{I}_{m}\left(u\left(x_{m}^{-}\right)\right)\right| .
\end{aligned}
$$

From the hypotheses $(A 2)$ and (A3), we can easily show that the right hand side of the above inequality tends to zero independently of $u$ as $t_{1} \rightarrow t_{2}$. From Step 1 to Step 3 together with the Arzelá-Ascoli theorem, we can conclude that $M: \mathfrak{P C}(\mathfrak{J}, \mathbb{R}) \rightarrow P(\mathfrak{P C}(\mathfrak{J}, \mathbb{R}))$ is completely continuous.

Step 4: $M$ has a closed graph. Let $u_{n} \rightarrow u_{*}, h_{n} \in M\left(u_{n}\right)$ and $h_{n} \rightarrow h_{*}$. we want to prove that $h_{*} \in M\left(u_{*}\right)$. $h_{n} \in M\left(u_{n}\right)$ means that there 
exists $g_{n} \in S_{H, u_{n}}$ such that, for each $x \in \mathfrak{J}$,

$$
\begin{aligned}
h_{n}(x) & =u_{0}+u_{1}\left(\frac{x^{\rho}}{\rho}\right)+\frac{\rho^{1-\omega}}{\Gamma(\omega)} \sum_{0<x_{m}<x} \int_{x_{m-1}}^{x_{m}}\left(x_{m}^{\rho}-s^{\rho}\right)^{\omega-1} s^{\rho-1} g_{n}(s) \mathrm{d} s \\
& +\frac{\rho^{2-\omega}}{\Gamma(\omega-1)} \sum_{0<x_{m}<x}\left(x^{\rho-1}-x_{m}^{\rho-1}\right) \int_{x_{m-1}}^{x_{m}}\left(x_{m}^{\rho}-s^{\rho}\right)^{\omega-2} s^{\rho-1} g_{n}(s) \mathrm{d} s \\
& +\frac{\rho^{1-\omega}}{\Gamma(\omega)} \int_{x_{m}}^{x}\left(x^{\rho}-s^{\rho}\right)^{\omega-1} s^{\rho-1} g_{n}(s) \mathrm{d} s \\
& +\sum_{0<x_{m}<x} I_{m}\left(u_{n}\left(x_{m}^{-}\right)\right)+\sum_{0<x_{m}<x}\left(x-x_{m}\right) \bar{I}_{m}\left(u_{n}\left(x_{m}^{-}\right)\right) .
\end{aligned}
$$

we want to prove that, there exists $g_{*} \in S_{H, u_{*}}$ such that, for each $x \in \mathfrak{J}$,

$$
\begin{aligned}
h_{*}(x) & =u_{0}+u_{1}\left(\frac{x^{\rho}}{\rho}\right)+\frac{\rho^{1-\omega}}{\Gamma(\omega)} \sum_{0<x_{m}<x} \int_{x_{m-1}}^{x_{m}}\left(x_{m}^{\rho}-s^{\rho}\right)^{\omega-1} s^{\rho-1} g_{*}(s) \mathrm{d} s \\
& +\frac{\rho^{2-\omega}}{\Gamma(\omega-1)} \sum_{0<x_{m}<x}\left(x^{\rho-1}-x_{m}^{\rho-1}\right) \int_{x_{m-1}}^{x_{m}}\left(x_{m}^{\rho}-s^{\rho}\right)^{\omega-2} s^{\rho-1} g_{*}(s) \mathrm{d} s \\
& +\frac{\rho^{1-\omega}}{\Gamma(\omega)} \int_{x_{m}}^{x}\left(x^{\rho}-s^{\rho}\right)^{\omega-1} s^{\rho-1} g_{*}(s) \mathrm{d} s \\
& +\sum_{0<x_{m}<x} I_{m}\left(u_{*}\left(x_{m}^{-}\right)\right)+\sum_{0<x_{m}<x}\left(x-x_{m}\right) \bar{I}_{m}\left(u_{*}\left(x_{m}^{-}\right)\right) .
\end{aligned}
$$

Since $H(x, \cdot)$ is upper semi continuous, then for every $\varepsilon>0$, there exist $m_{0}(\varepsilon) \geq 0$ such that for every $m \geq m_{0}$, we get

$$
g_{n}(x) \in H\left(x, u_{n}(x)\right) \subset H\left(x, u_{*}(x)\right)+\varepsilon B(0,1) \text {, a.e. } x \in \mathfrak{J} .
$$

Since $H(\cdot, \cdot)$ has compact values, then there exists a subsequence $g_{m_{n}}(\cdot)$ such that

$$
\begin{gathered}
g_{m_{n}}(\cdot) \rightarrow g_{*}(\cdot), \text { as } n \rightarrow \infty, \\
g_{*}(x) \in H\left(x, u_{*}(x)\right), \text { a.e. } x \in \mathfrak{J} .
\end{gathered}
$$

Using the concept that the functions $I_{m}$ and $\bar{I}_{m}, m=1,2, \ldots k$ are continuous, we can easily prove that $h_{*}$ and $g_{*}$ satisfy (3.6).

Step 5: A priori bounds on solutions. Let $u \in \mathfrak{P C}(\mathfrak{J}, \mathbb{R})$ be such that $u \in \mu M(u)$ for $\mu \in(0,1)$. Then there exists $g \in S_{H, u}$ such that, for each $x \in \mathfrak{J}$,

$$
\begin{aligned}
|u(x)| & \leq\left|u_{0}\right|+\left|\frac{u_{1}}{\rho}\right| T^{\rho}+\frac{\rho^{1-\omega}}{\Gamma(\omega)} \sum_{0<x_{m}<x} \int_{x_{m-1}}^{x_{m}}\left(x_{m}^{\rho}-s^{\rho}\right)^{\omega-1} s^{\rho-1} q(s) \Phi(|u(s)|) \mathrm{d} s \\
& +\frac{\rho^{2-\omega}}{\Gamma(\omega-1)} \sum_{0<x_{m}<x}\left(x^{\rho-1}-x_{m}^{\rho-1}\right) \int_{x_{m-1}}^{x_{m}}\left(x_{m}^{\rho}-s^{\rho}\right)^{\omega-2} s^{\rho-1} q(s) \Phi(|u(s)|) \mathrm{d} s \\
& +\frac{\rho^{1-\omega}}{\Gamma(\omega)} \int_{x_{m}}^{x}\left(x^{\rho}-s^{\rho}\right)^{\omega-1} s^{\rho-1} q(s) \Phi(|u(s)|) \mathrm{d} s \\
& +\sum_{0<x_{m}<x} \Phi^{*}(|u(s)|)+\sum_{0<x_{m}<x} \bar{\Phi}^{*}(|u(s)|) \\
& \leq\left|u_{0}\right|+\left|\frac{u_{1}}{\rho}\right| T^{\rho}+\frac{k T^{\rho \omega} q^{0}}{\rho^{\omega} \Gamma(\omega+1)} \Phi\left(\|u\|_{\infty}\right)+\frac{k T^{\rho \omega} q^{0}}{\rho^{\omega-1} \Gamma(\omega)} \Phi\left(\|u\|_{\infty}\right)+\frac{k T^{\rho \omega} q^{0}}{\rho^{\omega} \Gamma(\omega+1)} \Phi\left(\|u\|_{\infty}\right) \\
& +k \Phi^{*}\left(\|u\|_{\infty}\right)+k \bar{\Phi}^{*}\left(\|u\|_{\infty}\right) .
\end{aligned}
$$

Thus,

$$
\frac{\|u\|_{\infty}}{\left|u_{0}\right|+\left|\frac{u_{1}}{\rho}\right| T^{\rho}+a \Phi\left(\|u\|_{\infty}\right)+k \Phi^{*}\left(\|u\|_{\infty}\right)+k \bar{\Phi}^{*}\left(\|u\|_{\infty}\right)} \leq 1 .
$$

Then by $(A 4)$, there exists $N$ such that $\|u\|_{\infty} \neq N$. Let

$$
U=\left\{u \in \mathfrak{P C}(\mathfrak{J}, \mathbb{R}):\|u\|_{\infty}<N\right\} .
$$

The operator $M: \bar{U} \rightarrow P(\mathfrak{P C}(\mathfrak{J}, \mathbb{R}))$ is upper semi-continuous and completely continuous. From the choice of $U$, there is no $u \in \partial U$ such that $u \in \mu M(u)$ for some $\mu \in(0,1)$. From the concepts of the nonlinear alternative of Leray-Schauder type [24], we conclude that $M$ has a fixed point $u$ in $\bar{U}$ which is a solution of the problem (1.1). This completes the proof of the theorem. 


\section{The nonconvex case}

In this section, we discuss about the concepts for the existence of solutions for the problem (1.1), when the right hand side has a nonconvex value. Now, we adopt the concepts from Bressan and Colombo [25], Covitz and Nodler [23], and some existence results for nonconvex valued differential inclusion in $[5,21]$. We consider the following hypotheses for the next theorem:

(A6) $H: \mathfrak{J} \times \mathbb{R} \rightarrow Q_{c p}(\mathbb{R})$ has the property that $H(\cdot, y): \mathfrak{J} \rightarrow Q_{c p}(\mathbb{R})$ is measurable, convex valued and integrable bounded for each $y \in \mathbb{R}$. (A7) There exist constants $\ell^{*}, \bar{\ell}^{*}>0$ such that

$$
\begin{aligned}
& \left|I_{m}(y)-I_{m}(\bar{y})\right| \leq \ell^{*}|y-\bar{y}|, \text { for each } y, \bar{y} \in \mathbb{R} \text { and } m=1,2, \ldots k, \\
& \left|\bar{I}_{m}(y)-\bar{I}_{m}(\bar{y})\right| \leq \bar{\ell}^{*}|y-\bar{y}|, \text { for each } y, \bar{y} \in \mathbb{R} \text { and } m=1,2, \ldots k .
\end{aligned}
$$

Theorem 4.1. Assume that (A5)-(A7). If

$$
\left[\frac{k \tilde{l} T^{\rho \omega}}{\rho^{\omega} \Gamma(\omega+1)}+\frac{k \tilde{l} T^{\rho \omega}}{\rho^{\omega-1} \Gamma(\omega)}+\frac{\tilde{l} T^{\rho \omega}}{\rho^{\omega} \Gamma(\omega+1)}+k\left(\ell^{*}+T^{\rho} \bar{\ell}^{*}\right)\right]<1,
$$

where $\tilde{l}=\sup \{\tilde{l}(x): x \in \mathfrak{J}\}$, then (1.1) has one solution on $\mathfrak{J}$.

Proof. For each $u \in \mathfrak{P C}(\mathfrak{J}, \mathbb{R})$, the set $S_{H, u}$ is nonempty. From $(A 6)$ and (see [26, Theorem III.6], $H$ has a measurable selection. We shall prove that $M$ satisfies the assumptions of Lemma 2.3. The proof contains two steps.

Step 1: $M(u) \in Q_{c l}(\mathfrak{P C}(\mathfrak{J}, \mathbb{R}))$ for each $u \in \mathfrak{P C}(\mathfrak{J}, \mathbb{R})$. Let $\left(u_{n}\right)_{n \geq 0} \in M(u)$ such that $u_{n} \rightarrow \tilde{u}$ in $\mathfrak{P C}(\mathfrak{J}, \mathbb{R})$. Then, $\tilde{u} \in \mathfrak{P C}(\mathfrak{J}, \mathbb{R})$ and there exists $g_{n} \in S_{H, u}$ such that for each $x \in \mathfrak{J}$,

$$
\begin{aligned}
u_{n}(x) & =u_{0}+u_{1}\left(\frac{x^{\rho}}{\rho}\right)+\frac{\rho^{1-\omega}}{\Gamma(\omega)} \sum_{0<x_{m}<x} \int_{x_{m-1}}^{x_{m}}\left(x_{m}^{\rho}-s^{\rho}\right)^{\omega-1} s^{\rho-1} g_{n}(s) \mathrm{d} s \\
& +\frac{\rho^{2-\omega}}{\Gamma(\omega-1)} \sum_{0<x_{m}<x}\left(x^{\rho-1}-x_{m}^{\rho-1}\right) \int_{x_{m-1}}^{x_{m}}\left(x_{m}^{\rho}-s^{\rho}\right)^{\omega-2} s^{\rho-1} g_{n}(s) \mathrm{d} s \\
& +\frac{\rho^{1-\omega}}{\Gamma(\omega)} \int_{x_{m}}^{x}\left(x^{\rho}-s^{\rho}\right)^{\omega-1} s^{\rho-1} g_{n}(s) \mathrm{d} s \\
& +\sum_{0<x_{m}<x} I_{m}\left(u\left(x_{m}^{-}\right)\right)+\sum_{0<x_{m}<x}\left(x-x_{m}\right) \bar{I}_{m}\left(u\left(x_{m}^{-}\right)\right) .
\end{aligned}
$$

From $(A 5)$ and $H$ has compact values, we may pass to a subsequence if necessary to get that $g_{n}$ converges weakly to $g$ in $\mathfrak{L}_{w}^{1}(\mathfrak{J}, \mathbb{R})$ (the space endowed with the weak topology). A standard argument shows that $g_{n}$ converges strongly to $g \in S_{H, u}$. Then, for each $x \in \mathfrak{J}$,

$$
\begin{aligned}
u_{n}(x) \rightarrow \tilde{u}(x) & =u_{0}+u_{1}\left(\frac{x^{\rho}}{\rho}\right)+\frac{\rho^{1-\omega}}{\Gamma(\omega)} \sum_{0<x_{m}<x} \int_{x_{m-1}}^{x_{m}}\left(x_{m}^{\rho}-s^{\rho}\right)^{\omega-1} s^{\rho-1} g(s) \mathrm{d} s \\
& +\frac{\rho^{2-\omega}}{\Gamma(\omega-1)} \sum_{0<x_{m}<x}\left(x^{\rho-1}-x_{m}^{\rho-1}\right) \int_{x_{m-1}}^{x_{m}}\left(x_{m}^{\rho}-s^{\rho}\right)^{\omega-2} s^{\rho-1} g(s) \mathrm{d} s \\
& +\frac{\rho^{1-\omega}}{\Gamma(\omega)} \int_{x_{m}}^{x}\left(x^{\rho}-s^{\rho}\right)^{\omega-1} s^{\rho-1} g(s) \mathrm{d} s \\
& +\sum_{0<x_{m}<x} I_{m}\left(u\left(x_{m}^{-}\right)\right)+\sum_{0<x_{m}<x}\left(x-x_{m}\right) \bar{I}_{m}\left(u\left(x_{m}^{-}\right)\right) .
\end{aligned}
$$

So, $\tilde{u} \in M(u)$.

Step 2: There exists $\gamma<1$ such that $G_{d}(M(u), M(\bar{u})) \leq \gamma\|u-\bar{u}\|_{\infty}$ for each $u, \bar{u} \in \mathfrak{P} \mathfrak{C}(\mathfrak{J}, \mathbb{R})$. Let $u, \bar{u} \in \mathfrak{P C}(\mathfrak{J}, \mathbb{R})$ and $h_{1} \in M(u)$. Then there exists $g_{1}(x) \in H(x, u(x))$ such that for each $x \in \mathfrak{J}$,

$$
\begin{aligned}
h_{1}(x) & =u_{0}+u_{1}\left(\frac{x^{\rho}}{\rho}\right)+\frac{\rho^{1-\omega}}{\Gamma(\omega)} \sum_{0<x_{m}<x} \int_{x_{m-1}}^{x_{m}}\left(x_{m}^{\rho}-s^{\rho}\right)^{\omega-1} s^{\rho-1} g_{1}(s) \mathrm{d} s \\
& +\frac{\rho^{2-\omega}}{\Gamma(\omega-1)} \sum_{0<x_{m}<x}\left(x^{\rho-1}-x_{m}^{\rho-1}\right) \int_{x_{m-1}}^{x_{m}}\left(x_{m}^{\rho}-s^{\rho}\right)^{\omega-2} s^{\rho-1} g_{1}(s) \mathrm{d} s \\
& +\frac{\rho^{1-\omega}}{\Gamma(\omega)} \int_{x_{m}}^{x}\left(x^{\rho}-s^{\rho}\right)^{\omega-1} s^{\rho-1} g_{1}(s) \mathrm{d} s \\
& +\sum_{0<x_{m}<x} I_{m}\left(u\left(x_{m}^{-}\right)\right)+\sum_{0<x_{m}<x}\left(x-x_{m}\right) \bar{I}_{m}\left(u\left(x_{m}^{-}\right)\right) .
\end{aligned}
$$

From $(A 5)$, we get

$$
G_{d}(H(x, u(x)), H(x, \bar{u}(x))) \leq \tilde{l}(x)|u(x)-\bar{u}(x)| .
$$

Therefore, there exists $z \in H(x, \bar{u}(x))$ such that

$$
\left|g_{1}(x)-z\right| \leq \tilde{l}(x)|u(x)-\bar{u}(x)|, x \in \mathfrak{J} .
$$


Consider $U: \mathfrak{J} \rightarrow P(\mathbb{R})$ given by

$$
U(x)=\left\{z \in \mathbb{R}:\left|g_{1}(x)-z\right| \leq \tilde{l}(x)|u(x)-\bar{u}(x)|\right\} .
$$

Since the multivalued operator $V(x)=U(x) \cap H(x, \bar{u}(x))$ is measurable (See [He, Proposition III.4]), there exists a function $g_{2}(x)$ which is a measurable selection for $V$. So, $g_{2}(x) \in H(x, \bar{u}(x))$, and for each $x \in \mathfrak{J}$,

$$
\left|g_{1}(x)-g_{2}(x)\right| \leq \tilde{l}(x)|u(x)-\bar{u}(x)| .
$$

Now, we define for each $x \in \mathfrak{J}$,

$$
\begin{aligned}
h_{2}(x) & =u_{0}+u_{1}\left(\frac{x^{\rho}}{\rho}\right)+\frac{\rho^{1-\omega}}{\Gamma(\omega)} \sum_{0<x_{m}<x} \int_{x_{m-1}}^{x_{m}}\left(x_{m}^{\rho}-s^{\rho}\right)^{\omega-1} s^{\rho-1} g_{2}(s) \mathrm{d} s \\
& +\frac{\rho^{2-\omega}}{\Gamma(\omega-1)} \sum_{0<x_{m}<x}\left(x^{\rho-1}-x_{m}^{\rho-1}\right) \int_{x_{m-1}}^{x_{m}}\left(x_{m}^{\rho}-s^{\rho}\right)^{\omega-2} s^{\rho-1} g_{2}(s) \mathrm{d} s \\
& +\frac{\rho^{1-\omega}}{\Gamma(\omega)} \int_{x_{m}}^{x}\left(x^{\rho}-s^{\rho}\right)^{\omega-1} s^{\rho-1} g_{2}(s) \mathrm{d} s \\
& +\sum_{0<x_{m}<x} I_{m}\left(u\left(x_{m}^{-}\right)\right)+\sum_{0<x_{m}<x}\left(x-x_{m}\right) \bar{I}_{m}\left(u\left(x_{m}^{-}\right)\right) .
\end{aligned}
$$

Then, for each $x \in \mathfrak{J}$,

$$
\begin{aligned}
\left|h_{1}(x)-h_{2}(x)\right| & \leq \frac{\rho^{1-\omega}}{\Gamma(\omega)} \sum_{0<x_{m}<x} \int_{x_{m-1}}^{x_{m}}\left(x_{m}^{\rho}-s^{\rho}\right)^{\omega-1} s^{\rho-1}\left|g_{1}(s)-g_{2}(s)\right| \mathrm{d} s \\
& +\frac{\rho^{2-\omega}}{\Gamma(\omega-1)} \sum_{0<x_{m}<x}\left(x^{\rho-1}-x_{m}^{\rho-1}\right) \int_{x_{m-1}}^{x_{m}}\left(x_{m}^{\rho}-s^{\rho}\right)^{\omega-2} s^{\rho-1}\left|g_{1}(s)-g_{2}(s)\right| \mathrm{d} s \\
& +\frac{\rho^{1-\omega}}{\Gamma(\omega)} \int_{x_{m}}^{x}\left(x^{\rho}-s^{\rho}\right)^{\omega-1} s^{\rho-1}\left|g_{1}(s)-g_{2}(s)\right| \mathrm{d} s \\
& +\sum_{0<x_{m}<x}\left|I_{m}\left(u\left(x_{m}^{-}\right)\right)-I_{m}\left(\bar{u}\left(x_{m}^{-}\right)\right)\right|+\sum_{0<x_{m}<x}\left(x-x_{m}\right)\left|\bar{I}_{m}\left(u\left(x_{m}^{-}\right)\right)-\bar{I}_{m}\left(\bar{u}\left(x_{m}^{-}\right)\right)\right| \\
& \leq \frac{\tilde{l} \rho^{1-\omega}}{\Gamma(\omega)} \sum_{m=1}^{k} \int_{x_{m-1}}^{x_{m}}\left(x_{m}^{\rho}-s^{\rho}\right)^{\omega-1} s^{\rho-1}|u(s)-\bar{u}(s)| \mathrm{d} s \\
& +\frac{\tilde{l} \rho^{2-\omega}}{\Gamma(\omega-1)} \sum_{0<x_{m}<x}\left(x^{\rho-1}-x_{m}^{\rho-1}\right) \int_{x_{m-1}}^{x_{m}}\left(x_{m}^{\rho}-s^{\rho}\right)^{\omega-2} s^{\rho-1}|u(s)-\bar{u}(s)| \mathrm{d} s \\
& +\frac{\tilde{l} \rho^{1-\omega}}{\Gamma(\omega)} \int_{x_{m}}^{x}\left(x^{\rho}-s^{\rho}\right)^{\omega-1} s^{\rho-1}|u(s)-\bar{u}(s)| \mathrm{d} s \\
& +\sum_{m=1}^{k} \ell^{*}\left|u\left(x_{m}^{-}\right)-\bar{u}\left(x_{m}^{-}\right)\right|+\sum_{m=1}^{k} \overline{\ell^{*}}\left(x-x_{m}\right)\left|u\left(x_{m}^{-}\right)-\bar{u}\left(x_{m}^{-}\right)\right| \\
& \leq \frac{k \tilde{l} T^{\rho \omega}}{\rho^{\omega} \Gamma(\omega+1)}\|u-\bar{u}\|_{\infty}+\frac{k \tilde{l} T^{\rho \omega}}{\rho^{\omega-1} \Gamma(\omega)}\|u-\bar{u}\|_{\infty} \\
& +\frac{\tilde{l} T^{\rho \omega}}{\rho^{\omega} \Gamma(\omega+1)}\|u-\bar{u}\|_{\infty}+k \ell^{*}\|u-\bar{u}\|_{\infty}+k T^{\rho} \bar{\ell}^{*}\|u-\bar{u}\|_{\infty} .
\end{aligned}
$$

Thus,

$$
\left\|h_{1}-h_{2}\right\|_{\infty} \leq\left[\frac{k \tilde{l} T^{\rho \omega}}{\rho^{\omega} \Gamma(\omega+1)}+\frac{k \tilde{l} T^{\rho \omega}}{\rho^{\omega-1} \Gamma(\omega)}+\frac{\tilde{l} T^{\rho \omega}}{\rho^{\omega} \Gamma(\omega+1)}+k\left(\ell^{*}+T^{\rho} \bar{\ell}^{*}\right)\right]\|u-\bar{u}\|_{\infty} .
$$

By an similar relation obtained by interchanging the roles of $u$ and $\bar{u}$, we get

$$
G_{d}(M(u), M(\bar{u})) \leq\left[\frac{k \tilde{l} T^{\rho \omega}}{\rho^{\omega} \Gamma(\omega+1)}+\frac{k \tilde{l} T^{\rho \omega}}{\rho^{\omega-1} \Gamma(\omega)}+\frac{\tilde{l} T^{\rho \omega}}{\rho^{\omega} \Gamma(\omega+1)}+k\left(\ell^{*}+T^{\rho} \bar{\ell}^{*}\right)\right]\|u-\bar{u}\|_{\infty} .
$$

From (4.1), $M$ is a contraction and by Lemma 2.3, $M$ has a fixed point $u$ which is a solution to (1.1). Hence the proof is complete.

Now, we prove a result for problem (1.1) by using the concept of the nonlinear alternative of Leray-Schauder type [24] for single-valued maps with a selection theorem due to Bressan-Colombo for lower semi-continuous multivalued maps with decomposable values, also see [27] for multivalued maps with decomposable values and their properties.

Let $E$ be a subset of $[0, T] \times \mathbb{R} . E$ is $\mathfrak{L} \otimes \mathscr{B}$ measurable if $E$ belongs to the $\sigma$-algebra generated by all sets of the form $J \times \mathfrak{D}$, where $J$ is Lebesgue measurable in $[0, T]$ and $\mathfrak{D}$ is Borel measurable in $\mathbb{R}$. A subset $E$ of $\mathfrak{L}^{1}([0, T], \mathbb{R})$ is decomposable if for all $y, z \in E$ and $J \subset[0, T]$ measurable, $y \chi_{J}+z \chi_{[0, T]-J} \in E$, where $\chi$ stands for the characteristic function.

Let $F: Y \rightarrow P(Y)$ be a multivalued operator with nonempty closed values. $F$ is lower semi-continuous, if the set $\{t \in Y: F(t) \cap B \neq \emptyset\}$ is open for any open set $B$ in $Y$. 
Definition 4.2. Let $X$ be a separable metric space and let $M: X \rightarrow P\left(\mathfrak{L}^{1}([0, T], \mathbb{R})\right)$ be a multivalued operator, then M has Bressan-Colombo property if

(1) $M$ is lower semi-continuous;

(2) M has nonempty closed and decomposable values.

Let $H:[0, T] \times \mathbb{R} \rightarrow P(\mathbb{R})$ be a multivalued map with a nonempty compact values. Consider to $H$ the multivalued operator $\mathfrak{H}: \mathfrak{P C}([0, T], \mathbb{R}) \rightarrow$ $P\left(\mathfrak{L}^{1}([0, T], \mathbb{R})\right)$ by letting

$$
\mathfrak{H}(u)=\left\{z \in \mathfrak{L}^{1}([0, T], \mathbb{R}): z(x) \in H(x, u(x)) \text { for a.e. } x \in[0, T]\right\} .
$$

The operator $\mathfrak{H}$ is called the Niemytzki operator associated with $H$.

Definition 4.3. Let $H:[0, T] \times \mathbb{R} \rightarrow P(\mathbb{R})$ be a multivalued function with nonempty compact values. We say $H$ is of lower semi-continuous type if its associated Niemytzki operator $\mathfrak{H}$ is lower semi-continuous and has nonempty closed and decomposable values.

Now we present a selection theorem due to Bressan and Colombo [25].

Theorem 4.4. [25] Let $X$ be a separable metric space and let the operator $M: X \rightarrow P\left(\mathfrak{L}^{1}([0, T], \mathbb{R})\right)$ be a multivalued satisfying property Bressan and Colombo. Then $M$ has a continuous selection, that is there exists a continuous function(single-valued) $\tilde{f}: X \rightarrow \mathfrak{L}^{1}([0,1], \mathbb{R})$ such that $\tilde{f}(u) \in M(u)$ for every $u \in X$.

Next we introduce the following hypotheses:

(A8) $H:[0, T] \times \mathbb{R} \rightarrow P(\mathbb{R})$ is a nonempty compact valued multivalued map such that:

(a) $(x, u) \mapsto H(x, u)$ is $\mathfrak{L} \otimes \mathscr{B}$ measurable;

(b) $u \mapsto H(x, u)$ is lower semi-continuous for a.e. $x \in[0, T]$.

(A9) For each $p>0$, there exists a function $h_{p} \in \mathfrak{L}^{1}\left([0, T], \mathbb{R}^{+}\right)$such that

$$
\|H(x, u)\|_{P} \leq h_{p}(x) \text { for a.e. } x \in[0, T],
$$

and for $u \in \mathbb{R}$ with $|u| \leq p$.

The following lemma plays important role in our main result.

Lemma 4.5. [28] Let $H:[0, T] \times \mathbb{R} \rightarrow P(\mathbb{R})$ be a multivalued map with nonempty, compact values. Assume that (A8), (A9) hold, then $H$ is of lower semi-continuous.

Theorem 4.6. Suppose that hypotheses (A2)-(A4), (A8), and (A9) are satisfied. Then the problem (1.1) has at least one solution.

Proof. From hypotheses (A8), (A9) and Lemma 4.5, $F$ is of lower semi-continuous type. Then from Theorem 4.4, there exists a continuous function $h \in \mathfrak{P C}([0, T], \mathbb{R}) \rightarrow \mathfrak{L}^{1}([0, T], \mathbb{R})$ such that $h(u) \in \mathfrak{H}(u)$ for all $u \in \mathfrak{P C}([0, T], \mathbb{R})$. Consider the problem

$$
\begin{cases}{ }^{\rho} D_{0^{+}}^{\omega} u(x) \in h(u)(x), & \text { for a.e. } x \in \mathfrak{J}=[0, T], x \neq x_{k}, m=1,2, \ldots k, 1<\omega \leq 2, \\ \left.\Delta u\right|_{x=x_{m}}=I_{m}\left(u\left(x_{m}^{-}\right)\right), & m=1,2, \ldots, k, \\ \left.\Delta u^{\prime}\right|_{x=x_{m}}=\bar{I}_{m}\left(u\left(x_{m}^{-}\right)\right), & m=1,2, \ldots, k, \\ u(0)=u_{0}, u^{\prime}(0)=u_{1} . & \end{cases}
$$

If $u$ is a solution of (4.2), then $u$ is a solution of (1.1). Problem (4.2) can be reformulated as a fixed point problem for the operator $M_{1}: \mathfrak{P C}([0, T], \mathbb{R}) \rightarrow \mathfrak{P C}([0, T], \mathbb{R})$ defined by

$$
\begin{aligned}
M_{1}(u)(x) & =u_{0}+u_{1}\left(\frac{x^{\rho}}{\rho}\right)+\frac{\rho^{1-\omega}}{\Gamma(\omega)} \sum_{0<x_{m}<x} \int_{x_{m-1}}^{x_{m}}\left(x_{m}^{\rho}-s^{\rho}\right)^{\omega-1} s^{\rho-1} h(u)(s) \mathrm{d} s \\
& +\frac{\rho^{2-\omega}}{\Gamma(\omega-1)} \sum_{0<x_{m}<x}\left(x^{\rho-1}-x_{m}^{\rho-1}\right) \int_{x_{m-1}}^{x_{m}}\left(x_{m}^{\rho}-s^{\rho}\right)^{\omega-2} s^{\rho-1} h(u)(s) \mathrm{d} s \\
& +\frac{\rho^{1-\omega}}{\Gamma(\omega)} \int_{x_{m}}^{x}\left(x^{\rho}-s^{\rho}\right)^{\omega-1} s^{\rho-1} h(u)(s) \mathrm{d} s \\
& +\sum_{0<x_{m}<x} I_{m}\left(u\left(x_{m}^{-}\right)\right)+\sum_{0<x_{m}<x}\left(x-x_{m}\right) \bar{I}_{m}\left(u\left(x_{m}^{-}\right)\right) .
\end{aligned}
$$

Using (A2)-(A4) and from similar argument as in Theorem 3.5, we can prove that the operator $M_{1}$ satisfies all conditions in the Leray-Schauder alternative.

\section{Topological structure of the solution set}

In this section, we present a theorem on the topological structure of the set of solutions to (1.1).

Theorem 5.1. Assume that (A1), (A5) and the following hypotheses hold:

(A10) There exists $q_{1} \in \mathfrak{C}\left(\mathfrak{J}, \mathbb{R}^{+}\right)$such that $\|H(x, y)\|_{P} \leq q_{1}(x)$ for $x \in \mathfrak{J}$ and $y \in \mathbb{R}$.

(A11) There exists $d_{1}, d_{2}>0$ such that

$$
\begin{aligned}
& \left|I_{m}(y)\right| \leq d_{1}, \text { for } y \in \mathbb{R}, \\
& \left|\bar{I}_{m}(y)\right| \leq d_{2}, \text { for } y \in \mathbb{R} .
\end{aligned}
$$


Then the solution set of (1.1) is not empty and is compact in $\mathfrak{P C}(\mathfrak{J}, \mathbb{R})$.

Proof. Let

$$
S=\{u \in \mathfrak{P C}(\mathfrak{J}, \mathbb{R}): u \text { is a solution of }(1.1)\}
$$

From Theorem 3.5, $S \neq \emptyset$. Now, we want to prove that $S$ is compact. Let $\left(u_{n}\right)_{n \in \mathbb{N}} \in S$, then there exists $g_{n} \in S_{H, u_{n}}$ and $x \in \mathfrak{J}$ such that

$$
\begin{aligned}
u_{n}(x) & =u_{0}+u_{1}\left(\frac{x^{\rho}}{\rho}\right)+\frac{\rho^{1-\omega}}{\Gamma(\omega)} \sum_{0<x_{m}<x} \int_{x_{m-1}}^{x_{m}}\left(x_{m}^{\rho}-s^{\rho}\right)^{\omega-1} s^{\rho-1} g_{n}(s) \mathrm{d} s \\
& +\frac{\rho^{2-\omega}}{\Gamma(\omega-1)} \sum_{0<x_{m}<x}\left(x^{\rho-1}-x_{m}^{\rho-1}\right) \int_{x_{m-1}}^{x_{m}}\left(x_{m}^{\rho}-s^{\rho}\right)^{\omega-2} s^{\rho-1} g_{n}(s) \mathrm{d} s \\
& +\frac{\rho^{1-\omega}}{\Gamma(\omega)} \int_{x_{m}}^{x}\left(x^{\rho}-s^{\rho}\right)^{\omega-1} s^{\rho-1} g_{n}(s) \mathrm{d} s \\
& +\sum_{0<x_{m}<x} I_{m}\left(u_{n}\left(x_{m}^{-}\right)\right)+\sum_{0<x_{m}<x}\left(x-x_{m}\right) \bar{I}_{m}\left(u_{n}\left(x_{m}^{-}\right)\right) .
\end{aligned}
$$

From hypotheses $(A 1),(A 10)$ and $(A 11)$, we can show that there exists an $N_{1}>0$ such that $\left\|u_{n}\right\|_{\infty} \leq N_{1}$ for every $n \geq 1$. As in Step 3 in Theorem 3.5, we can prove that the set $\left\{u_{n}: n \geq 1\right\}$ is equicontinuous in $\mathfrak{P C}(\mathfrak{J}, \mathbb{R})$. By Arzelá-Ascoli theorem, we can say that, there exists a subsequence (denoted again by $\left.\left\{u_{n}\right\}\right)$ of $\left\{u_{n}\right\}$ such that $u_{n}$ converges to $u$ in $\mathfrak{P C}(\mathfrak{J}, \mathbb{R})$. We shall prove that there exist $g(\cdot) \in F(\cdot, u(\cdot))$ and $x \in \mathfrak{J}$ such that

$$
\begin{aligned}
u(x) & =u_{0}+u_{1}\left(\frac{x^{\rho}}{\rho}\right)+\frac{\rho^{1-\omega}}{\Gamma(\omega)} \sum_{0<x_{m}<x} \int_{x_{m-1}}^{x_{m}}\left(x_{m}^{\rho}-s^{\rho}\right)^{\omega-1} s^{\rho-1} g(s) \mathrm{d} s \\
& +\frac{\rho^{2-\omega}}{\Gamma(\omega-1)} \sum_{0<x_{m}<x}\left(x^{\rho-1}-x_{m}^{\rho-1}\right) \int_{x_{m-1}}^{x_{m}}\left(x_{m}^{\rho}-s^{\rho}\right)^{\omega-2} s^{\rho-1} g(s) \mathrm{d} s \\
& +\frac{\rho^{1-\omega}}{\Gamma(\omega)} \int_{x_{m}}^{x}\left(x^{\rho}-s^{\rho}\right)^{\omega-1} s^{\rho-1} g(s) \mathrm{d} s \\
& +\sum_{0<x_{m}<x} I_{m}\left(u\left(x_{m}^{-}\right)\right)+\sum_{0<x_{m}<x}\left(x-x_{m}\right) \bar{I}_{m}\left(u\left(x_{m}^{-}\right)\right) .
\end{aligned}
$$

Since $H(x, \cdot)$ is upper semi-continuous, for every $\varepsilon>0$, there exists $n_{0}(\varepsilon) \geq 0$ such that for every $n \geq n_{0}$, we get

$$
g_{n}(x) \in H\left(x, u_{n}(x)\right) \subset H(x, u(x))+\varepsilon B(0,1) \text {, a.e. } x \in \mathfrak{J} .
$$

Since $H(\cdot, \cdot)$ has compact values, there exists subsequence $g_{n_{m}}(\cdot)$ such that

$$
\begin{gathered}
g_{n_{m}}(\cdot) \rightarrow g(\cdot) \text { as } m \rightarrow \infty, \\
g(x) \in H(x, u(x)), \text { a.e. } x \in \mathfrak{J} .
\end{gathered}
$$

Therefore,

$$
\left|g_{n_{m}}(x)\right| \leq q_{1}(x) \text {, a.e. } x \in \mathfrak{J}
$$

By Lebesgue's dominated convergence theorem, we say that $g \in \mathfrak{L}^{1}(\mathfrak{J}, \mathbb{R})$ which implies that $g \in S_{H, u}$. Thus, for $x \in \mathfrak{J}$, we get

$$
\begin{aligned}
u(x) & =u_{0}+u_{1}\left(\frac{x^{\rho}}{\rho}\right)+\frac{\rho^{1-\omega}}{\Gamma(\omega)} \sum_{0<x_{m}<x} \int_{x_{m-1}}^{x_{m}}\left(x_{m}^{\rho}-s^{\rho}\right)^{\omega-1} s^{\rho-1} g(s) \mathrm{d} s \\
& +\frac{\rho^{2-\omega}}{\Gamma(\omega-1)} \sum_{0<x_{m}<x}\left(x^{\rho-1}-x_{m}^{\rho-1}\right) \int_{x_{m-1}}^{x_{m}}\left(x_{m}^{\rho}-s^{\rho}\right)^{\omega-2} s^{\rho-1} g(s) \mathrm{d} s \\
& +\frac{\rho^{1-\omega}}{\Gamma(\omega)} \int_{x_{m}}^{x}\left(x^{\rho}-s^{\rho}\right)^{\omega-1} s^{\rho-1} g(s) \mathrm{d} s \\
& +\sum_{0<x_{m}<x} I_{m}\left(u\left(x_{m}^{-}\right)\right)+\sum_{0<x_{m}<x}\left(x-x_{m}\right) \bar{I}_{m}\left(u\left(x_{m}^{-}\right)\right) .
\end{aligned}
$$

Then,

$$
S \in P_{c p}(\mathfrak{P C}(\mathfrak{J}, \mathbb{R}))
$$




\section{An example}

Example 6.1. We consider the Katugampola fractional impulsive differential inclusions of the type,

$$
\left\{\begin{array}{l}
{ }^{\rho} D_{0^{+}}^{\omega} u(x) \in H(x, u(x)), \quad \text { a. e. } x \in \mathfrak{J}=[0, T], x \neq \frac{1}{3}, 1<\omega \leq 2, \\
\left.\Delta u\right|_{x=\frac{1}{3}}=\frac{1}{6+\left|u\left(\frac{1}{3}^{-}\right)\right|}, \\
\left.\Delta u^{\prime}\right|_{x=\frac{1}{3}}=\frac{1}{8+\left|u\left(\frac{1^{-}}{3}\right)\right|} \\
u(0)=u_{0}, u^{\prime}(0)=0
\end{array}\right.
$$

Let us assume the values $T=1, k=1, x_{1}=\frac{1}{3}, \rho=1$, and $x_{0}=x_{1}=0$. Set

$$
H(x, u)=\left\{g \in \mathbb{R}: f_{1}(x, u) \leq g \leq f_{2}(x, u)\right\},
$$

where the functions $f_{1}, f_{2}: \mathfrak{J} \times \mathbb{R} \rightarrow \mathbb{R}$ are given.

$$
I_{1}\left(u\left(x_{1}\right)\right)=\frac{1}{6+\left|u\left(\frac{1}{3}^{-}\right)\right|}, \bar{I}_{1}\left(u\left(x_{1}\right)\right)=\frac{1}{8+\left|u\left(\frac{1}{3}^{-}\right)\right|} .
$$

Then the eqaution (6.1) takes the form (1.1). We consider for each $x \in \mathfrak{J}$, the function $f_{1}(x, \cdot)$ is lower semi-continuous(i.e.,the set $\{u \in \mathbb{R}$ : $\left.f_{1}(x, u)>\lambda\right\}$ is open for each $\lambda \in \mathbb{R}$ ), and assume that for each $x \in \mathfrak{J}, f_{2}(x, \cdot)$ is upper semi-continuous(i.e.,the set $\left\{u \in \mathbb{R}: f_{2}(x, u)<\lambda\right\}$ is open for each $\lambda \in \mathbb{R})$. Assume that there are $q \in \mathfrak{C}\left(\mathfrak{J}, \mathbb{R}^{+}\right)$and $\Phi:[0, \infty) \rightarrow(0, \infty)$ continuous and non decreasing such that

$$
\max \left(\left|f_{1}(x, u)\right|,\left|f_{2}(x, u)\right|\right) \leq q(x) \Phi(|u|), x \in \mathfrak{J} \text { and } u \in \mathbb{R} .
$$

Assume that there exists a constant $N>0$ such that

$$
\frac{N}{\left(\frac{2 q^{0}}{\Gamma(\omega+1)}+\frac{q^{o}}{\Gamma(\omega)}\right) \Phi(N)+\frac{7}{24}}>1 .
$$

From this, $H$ is compact and convex valued and it is upper semi-continuous [20]. Since all the conditions of Theorem 3.5 are satisfied, the problem (6.1) has at least one solution $u$ on $\mathfrak{J}$.

\section{Conclusion}

In this article, Leray-Schauder type, Bressan and Colombo, Covitz and Nodler concepts are used to prove the the Katugampola fractional type impulsive differential equations with inclusions. The obtained conditions ensure that the existence of at least one solution to the proposed problem. Further, an example is investigated for the problem.

\section{Acknowledgement}

The authors are very grateful to thank referees for their careful reading of the manuscript and valuable comments which improved the quality of the paper. The authors thank the help from editor too.

\section{References}

[1] R. Hilfer, Applications of Fractional Calculus in Physics, World Scientific, Singapore, 2000.

[2] A. A. Kilbas, H. M. Srivastava, J. J. Trujillo, Theory and Applications of Fractional Differential Equations, Elsevier Science B.V., Amsterdam, 2006.

[3] I. Podlubny, Fractional Differential Equations, Academic Press, San Diego, 1999.

[4] R. P. Agarwal, M. Benchohra, S. Hamani, Boundary value problems for differential inclusions with fractional order, Adv. Cont. Math., 12(2) (2008), $181-196$.

[5] J.P. Aubin, A. Cellina, Differential Inclusions, Springer-Verlag, Berlin-Heidelberg, New York, 1984.

[6] M. Benchohra, S. Hamani, Nonlinear boundary value problems for differential Inclusions with Caputo fractional derivative, Topol. Methods Nonlinear Anal., 321 (2008), 115-130.

[7] K. Diethelm, N. J. Ford, Analysis of fractional differential equations, J. Math. Anal.Appl., 265 (2002), 229-248.

[8] A. A. Kilbas, S. A. Marzan, Nonlinear differential equations with the Caputo fractional derivative in the space of continuously differentiable functions, J. Differential Equations, 41 (2005), 84-89.

[9] D. Vivek, K. Kanagarajan, S. Harikrishnan, Theory and analysis of impulsive type pantograph equations with Katugampola fractional derivative, J. Vibration Testing and System Dynamics, 2(1) (2018), 9-20.

[10] U. N. Katugampola, Existence and uniqueness results for a class of generalized fractional differential equations, (2016), arXiv:1411.5229v2[math.CA].

[11] U. N. Katugampola, A new approach to generalized fractional derivatives, Bull. Math. Anal. Appl., 6(4) (2014), 1-15.

[12] U. N. Katugampola, New approach to a generalized fractional integral, Appl. Math. Comput., 218(3) (2011), 860-865.

[13] M. Benchohra, B. A. Slimani, Impulsive fractional differential equations, Electron. J. Differ. Equ., 10 (2009), 1-11.

[14] J. R. Graef, J. Henderson, A. Ouahab, Impulsive Differential Inclusions: A Fixed Point Approach, De Gruyter, Berlin/Boston, 2013.

[15] J. Henderson, J. Ouahab, Impulsive differential inclusions with fractional order, Comput. Math. Appl., 59 (2010), 1191-1226.

[16] V. Lakshmikantham, D. D. Bainov, P. S. Simeonov, Theory of Impulsive Differential Equations, World Scientific, Singapore, 1989.

[17] E. E. Ndiyo, Existence result for solution of second order impulsive differential inclusion to dynamic evolutionary process, Amer. J. Appl. Math., 7(2) (2017), 89-92.

[18] M. Benchohra, J. Henderson, S. K. Ntouyas, Impulsive Differential Equations and Inclusions, Hindawi Publishing Corporation, 2, New York, 2006.

[19] M. Benchohra, J. J. Nieto, B. A. Slimani, Existence of solutions to differential inclusions with fractional order and impulses, Electron. J. Differ. Equ., 80 (2010), 1-18.

[20] K. Deimling, Multivalued Differential Equations, Walter De Gruyter, Berlin-New York, 1992.

[21] Sh. Hu, N. Papageorgiou, Handbook of Multivalued Analysis, Theory I, Kluwer, Dordrecht, 1997.

[22] M. Kisielewicz, Differential Inclusions and Optimal Control, Kluwer, Dordrecht, The Netherlands, 1991.

[23] H. Covitz, S. B. Nadler Jr, Multivalued contraction mappings in generalized metric spaces, Israel J. Math., 8 (1970), 5-11. 
[24] A. Granas, J. Dugundji, Fixed Point Theory, Springer-Verlag, New York, 2003.

[25] A. Bressan, G. Colombo, Extensions and selections of maps with decomposable values, Studia Math., 90 (1988), 69-86.

[26] C. Castaing, M. Valadier, Convex Analysis and Measurable Multifunctions, Lecture Notes in Mathematics 580, Springer-Verlag, Berlin-Heidelberg-New York, 1977.

[27] A. Fryszkowski, Fixed Point Theory for Decomposable Sets, Topol. Fixed Point Theory Appl., 2. Kluwer Academic Publishers, Dordrecht, 2004.

[28] M. Frigon, A. Granas, Théorèmes d'existence pour des inclusions différentielles sans convexitè, Comptes Rendus Acad. Sci., Paris, Ser. I, 310 (1990), $819-822$ 